\title{
Determinants of knowledge and prevention measures towards COVID-19 pandemic among Lebanese dentists: a cross sectional survey
}

\author{
Mira Hleyhel ${ }^{1,2,3^{*}{ }^{+}}$, Christine Haddad ${ }^{3 \dagger}$, Nour Haidar ${ }^{3}$, Maria Charbachy ${ }^{3}$ and Nadine Saleh ${ }^{1,2,3}$
}

\begin{abstract}
Background: Coronavirus Disease 2019 (COVID-19) is a major global threat. Healthcare professionals including dentists are facing real challenges during this pandemic. This study aimed to evaluate knowledge, attitudes, and prevention measures of Lebanese dentists towards COVID-19 and determinants of high level of knowledge and prevention practices.

Methods: A cross-sectional study was conducted between May and August 2020 in Lebanon on a random sample of 323 Lebanese dentists. Data were collected through an online survey questionnaire. A multivariate linear regression model was used to evaluate factors associated with COVID-19 knowledge. A multivariate logistic regression was conducted to evaluate the factors associated with high level of prevention measures towards COVID-19.

Results: The mean COVID-19 knowledge index was 24.5 over 38 with only 15\% achieving high knowledge level. The mean prevention measures index was 11.4 over 16 with only $35 \%$ achieving high prevention level. Higher knowledge index was associated with younger age, being employed, and considering dentist's role significant in teaching others about COVID-19. General dental practitioners, dentists living with family members and concerned about their family members to get infected because of their occupational exposure were more likely to report higher level of adopted prevention measures. Higher knowledge was associated with high level of prevention measures.
\end{abstract}

Conclusions: Given the rapid evolution of information related to COVID-19 pandemic, dentists should be regularly educated through trainings, workshops, and updates of national guidelines for dental healthcare.

Keywords: SARS-CoV-2, Health knowledge, Prevention, Dental practice, Pandemic

\section{Introduction}

The World Health Organization has declared Coronavirus Disease 2019 (COVID-19) a pandemic on March 11, 2020 , considering it a worldwide public health emergency

\footnotetext{
*Correspondence: mira.hleyhel@hotmail.com

${ }^{\dagger}$ Mira Hleyhel and Christine Haddad have contributed equally to the work

${ }^{1}$ CERIPH, Center for Research in Public Health, Pharmacoepidemiology

Surveillance Unit, Faculty of Public Health II, Lebanese University, Fanar,

Lebanon

Full list of author information is available at the end of the article
}

$[1,2]$. COVID-19 is caused by a novel coronavirus, called severe acute respiratory syndrome coronavirus 2 (SARSCoV-2). As of February 2021, the worldwide global cumulative number of cases was estimated at 105.4 million with 2.3 million cases of deaths [3]. In Lebanon, as of February 15, 2021, and since the start of the pandemic, an estimated 340,861 SARS-CoV-2 cases and 4037 deaths were recorded with a fatality rate of $1.2 \%$ [4].

Until now, there is still no universal effective treatment acting specifically against COVID-19 [5]. For this, 
adopting the appropriate preventive measures remains one of the best solutions to fight against the infection. Since the emergence of SARS-CoV-2, healthcare professionals have been facing critical challenges. Dental professionals who are in close contact with the patient, are at great risk of exposure to the virus, while performing procedures and surgical acts that produce a mixture of splatter, droplets, and aerosols. Thus, it is important to evaluate dentists' knowledge and prevention measures towards COVID-19 as it is highly likely that the current pandemic will impact dental practice in general and will shape the training of the future generations of dentists. A contribution to the fight against the pandemic is expected from dentists through spreading awareness, screening for suspected cases, providing consultations and through updating dentistry curricula to include trainings about new methods of prevention control and about preparedness for future pandemics [6].

To control the spread of the virus in dental healthcare facilities, the Centers for Disease Control and Prevention [7, 8], and the American Dental Association [9] have published different guidelines for dental healthcare practice. In Lebanon, dental healthcare guidelines [10] had been released by the Lebanese Dental Association (LDA) at the time when data started to be collected for the study. Those guidelines highlighted the types of personal protective equipment (PPE) required and their appropriate use, the definition of a dental emergency, and included a protocol for dental care during COVID-19 pandemic.

COVID-19, which is a highly contagious disease, constitutes a major threat to the health of dentists, their assistants, and patients. Therefore, dentists should have information and should be aware of the most effective prevention measures to protect their owns and patients' health. Given their crucial role in combatting the outbreak of the disease, we aimed in this study to assess the knowledge, attitudes, prevention measures and clinical practices of Lebanese dentists regarding COVID-19 pandemic.

\section{Methods}

\section{Study design and sampling}

A cross-sectional survey was conducted from May to August 2020 using a web-based questionnaire. A stratified random sampling from the list of all Lebanese dentists registered in the LDA was conducted. For this study, $10 \%$ of dentists' population was taken as sample size estimated at 320 dentists. The sample was proportionally distributed according to the Lebanese governorates (Beirut, Mount Lebanon, North Lebanon, South Lebanon and Bekaa). Assuming that the rate of completed questionnaires will be $20 \%$ [11], the total number of dentists to be selected and invited to participate were 1600 dentists.

\section{Questionnaire and data collection}

The questionnaire was designed in English and then translated to French and reviewed for consistency. The questions were developed based on the literature and international guidelines. The questionnaire included:

1. Sociodemographic characteristics and factors related to participants general practices and health.

2. Knowledge assessed by 7 questions including 38 answers on: clinical symptoms, incubation period, modes of transmission, molecules under investigation in clinical trials and mouthwash virucidal activity against the COVID-19 virus.

3. Prevention measures adopted in dental clinics and other practices.

4. Attitudes towards COVID-19 pandemic

Each participant was contacted by phone call where the survey objective was described. After obtaining an oral informed consent from the participant, a google form link was sent through phone message or whatsapp.

\section{Statistical analysis}

Descriptive analysis was used to describe participant characteristics using means and standard deviations (SD) for quantitative variables and frequencies and percentages for qualitative variables. For knowledge index, a correct answer was given one point and wrong or "I don't know" was given zero point. The total knowledge index was calculated by summing all the answers and ranged between 0 and 38. The index was classified into 3 levels of knowledge, high $(80-100 \%)$, moderate $(60-79 \%)$ or low $(\leq 59 \%)$ level [12]. The same method was used to calculate and categorize the prevention measures index which ranged between 0 and 16 .

A multivariate linear regression model was performed to identify factors associated with the knowledge index. A multivariate logistic regression model was used to explore factors associated with high prevention measures level taking low and moderate index combined as the reference category. Independent variables associated with the dependent variables with a p-value $\leq 0.2$ in the bivariate analyses were included in the multivariate models [13]. Data was analyzed using SPSS software.

\section{Results}

\section{Characteristics of the study participants}

A total of 323 dentists completed the questionnaire. The age of the participants ranged between 25 and 70 years 
old, with more than half (57.3\%) being males. Around a quarter of the participants were general dental practitioners, most of them had more than 10 years of experience, and only $17.6 \%$ had comorbidities. A detailed description of study participants characteristics is presented in Table 1.

\section{Knowledge towards COVID-19}

Almost all the respondents reported the main COVID-19 symptoms which included fever (99.1\%), fatigue (91.3\%), dry cough $(92.6 \%)$ and headache $(92.9 \%)$. However less than half reported the less common symptoms. Most of the dentists (73\%) reported the correct average incubation period of the virus being 1-14 days and identified the modes of transmission of the virus. Regarding molecules under investigation in clinical trials, $90.7 \%$ of the respondents reported hydroxychloroquine, meanwhile, less than half of the respondents reported other molecules (Table 2). The mean knowledge index regarding COVID-19 was $64.5 \%$ and its Cronbach alpha for its internal consistency was 0.79 . Low and moderate knowledge was observed in around $85 \%$ of the participants (Table 3).

\section{Prevention measures towards COVID-19}

Almost all the respondents reported adopting the right preventive measures in the dental clinics by applying hand hygiene (95.7\%), disinfection for surfaces (90.4\%) use of PPE (93.2\%) and protective clothes for staff members $(84.8 \%)$. More than half of the respondents reported that they use high-speed evacuation for dental procedures (65\%), avoid operations that can produce droplets or aerosols (57.6\%) and avoid moving patients out of their area unless necessary $(54.8 \%)$. Only $30.7 \%$ of respondents reported placing patients with known or suspected COVID-19 infection in adequately ventilated single rooms (Table 4a).

Most dentists reported adopting the right prevention measures with the patients, by asking them to sit far from each others $(77.1 \%)$, wearing masks $(97.2 \%)$, applying hand hygiene $(88.2 \%)$, screening patients $(70.9 \%)$, taking their temperature (91\%) and asking about recent travel (62.8\%). Meanwhile, less than half of the respondents reported asking patients about recent contact with suspected or known infected people $(42.7 \%)$ and the use of rubber dam for dental procedures (37.8\%) (Table 4b).

Overall, the mean prevention measures index of the participants was $71.2 \%$ and the Cronbach alpha for its internal consistency was 0.67 . While $21.1 \%$ of dentists reported adopting low prevention measures level, 34.7\% reported adopting high level of prevention measures towards COVID-19 (Table 3).
Table 1 Characteristics of study participants $(n=323)$

\begin{tabular}{|c|c|c|}
\hline Characteristics & Mean \pm SD & $\mathrm{N}(\%)$ \\
\hline Age (years) & $44.62 \pm 10.8$ & \\
\hline \multicolumn{3}{|l|}{ Gender } \\
\hline Male & & $185(57.3 \%)$ \\
\hline Female & & $138(42.7 \%)$ \\
\hline \multicolumn{3}{|l|}{ Work region } \\
\hline Beirut & & $64(19.8 \%)$ \\
\hline Mount Lebanon & & $178(55.1 \%)$ \\
\hline Bekaa, South and North of Lebanon & & $81(25.1 \%)$ \\
\hline \multicolumn{3}{|l|}{ Graduation university } \\
\hline Public: LU & & $117(36.2 \%)$ \\
\hline Private: USJ or BAU & & $112(34.7 \%)$ \\
\hline Abroad & & $94(29.1 \%)$ \\
\hline \multicolumn{3}{|l|}{ Years of experience* } \\
\hline$\leq 10$ & & $88(27.2 \%)$ \\
\hline$>10$ & & $231(71.5 \%)$ \\
\hline \multicolumn{3}{|l|}{ Specialty $(1)^{\dagger}$} \\
\hline General practitioner & & $155(48 \%)$ \\
\hline Oral surgeon & & $64(19.8 \%)$ \\
\hline Implant surgeon & & $43(13.3 \%)$ \\
\hline Orthodontist & & $30(9.3 \%)$ \\
\hline Endodontic & & $29(9 \%)$ \\
\hline Periodontic & & $25(7.7 \%)$ \\
\hline Prosthodontic & & $22(6.8 \%)$ \\
\hline Restorative Dentist & & $18(5.6 \%)$ \\
\hline Other & & $21(6.5 \%)$ \\
\hline \multicolumn{3}{|l|}{ Specialty (2) } \\
\hline General practitioner only & & $84(26 \%)$ \\
\hline Surgeon (oral, implant) & & $104(32.2 \%)$ \\
\hline Specialist dentist & & $135(41.8 \%)$ \\
\hline \multicolumn{3}{|l|}{ Employment status } \\
\hline Self-employed & & $221(68.4 \%)$ \\
\hline Employee & & $95(29.4 \%)$ \\
\hline Unemployed & & $7(2.2 \%)$ \\
\hline \multicolumn{3}{|l|}{ Living with family members } \\
\hline Yes & & $233(72.1 \%)$ \\
\hline No & & $90(27.9 \%)$ \\
\hline \multicolumn{3}{|l|}{ Comorbidities $^{\dagger}$} \\
\hline None & & $268(83 \%)$ \\
\hline Hypertension & & $20(6.2 \%)$ \\
\hline Diabetes & & $18(5.6 \%)$ \\
\hline Cardiovascular disease & & $9(2.8 \%)$ \\
\hline Obesity & & $10(3.1 \%)$ \\
\hline Respiratory disease & & $5(1.5 \%)$ \\
\hline Active cancer & & $2(0.6 \%)$ \\
\hline Immunodeficiency & & $5(1.5 \%)$ \\
\hline
\end{tabular}

${ }^{*}$ Years of experience: missing data $=4$

${ }^{\dagger}$ The sum of the percentages is greater than $100 \%$ because dentists can have several specialties and several comorbidities

LU Lebanese University, USJ Saint-Joseph University, BAU Beirut Arab University 
Table 2 Responses to questions about Coronavirus Disease 2019 (COVID-19) knowledge by the participating dentists

\begin{tabular}{|c|c|c|}
\hline & Answers & $\mathrm{N}(\%)$ \\
\hline \multicolumn{3}{|l|}{ Incubation period (days) } \\
\hline $1-14$ & Correct & $238(73.7)$ \\
\hline $2-7$ & Incorrect & $28(8.7)$ \\
\hline $7-14$ & Incorrect & $25(7.7)$ \\
\hline $7-21$ & Incorrect & $25(7.7)$ \\
\hline I don't know & Incorrect & $7(2.2)$ \\
\hline \multicolumn{3}{|l|}{ Symptoms } \\
\hline Fever & Correct & $320(99.1)$ \\
\hline Fatigue & Correct & $295(91.3)$ \\
\hline Dry cough & Correct & $299(92.6)$ \\
\hline Headache & Correct & $300(92.6)$ \\
\hline Shortness of breath & Correct & 296 (91.6) \\
\hline Diarrhea & Correct & $155(48)$ \\
\hline Vomiting & Correct & $134(41.5)$ \\
\hline Runny nose & Correct & $161(49.8)$ \\
\hline Sore throat & Correct & $240(74.3)$ \\
\hline Red eyes & Correct & $98(30.3)$ \\
\hline Skin rash & Correct & $137(42.4)$ \\
\hline Joint or muscle pain & Correct & $197(61)$ \\
\hline Loss of smell and taste & Correct & $263(81.4)$ \\
\hline May present with no symptoms & Correct & $308(95.4)$ \\
\hline I don't know & Incorrect & $1(0.3)$ \\
\hline \multicolumn{3}{|l|}{ Mode of transmission } \\
\hline Droplets & Correct & $310(96)$ \\
\hline Coughing and sneezing & Correct & $319(98.8)$ \\
\hline Hand shaking & Correct & $264(81.7)$ \\
\hline Touching surfaces such as doorknobs and tables & Correct & $257(79.6)$ \\
\hline Contact with blood & Incorrect & $169(52.3)$ \\
\hline Contact with stools & Incorrect & $71(22)$ \\
\hline I don't know & Incorrect & $1(0.3)$ \\
\hline \multicolumn{3}{|l|}{ Transmission is possible when } \\
\hline Symptomatic & Correct & $300(92.9)$ \\
\hline Asymptomatic & Correct & $217(67.2)$ \\
\hline Asymptomatic with positive PCR & Correct & $300(92.9)$ \\
\hline I don't know & Incorrect & $7(2.2)$ \\
\hline \multicolumn{3}{|l|}{ Molecules under investigation in clinical trials } \\
\hline Chloroquine & Correct & $156(48.3)$ \\
\hline Hydroxychloroquine & Correct & $293(90.7)$ \\
\hline Azithromycin & Correct & $100(31)$ \\
\hline Ivermectin & Correct & $49(15.2)$ \\
\hline Remdesivir & Correct & $124(38.4)$ \\
\hline Lopinavir & Correct & $92(28.5)$ \\
\hline Ritonavir & Correct & $89(27.6)$ \\
\hline Fluoroquinolones & Incorrect & $12(3.7)$ \\
\hline Methylprednisolone & Correct & $9(2.8)$ \\
\hline Penicillin & Incorrect & $10(3.1)$ \\
\hline Oseltamivir & Incorrect & $11(3.4)$ \\
\hline Thalidomide & Correct & $6(1.9)$ \\
\hline I don't know & Incorrect & $14(4.3)$ \\
\hline
\end{tabular}


Table 2 (continued)

\begin{tabular}{lll}
\hline & Answers & N (\%) \\
\hline COVID-19 symptoms often resolve with time and do not require any special treatment & & 250 (77.4) \\
True & Correct & $58(18)$ \\
False & Incorrect & $15(4.6)$ \\
I don't know & Incorrect & \\
All mouthwash has a high virucidal activity against COVID-19 & Incorrect & 99(30.7) \\
True & Incorrect & 135) \\
False & Correct & 139(43) \\
There is a lack of studies & Incorrect & $30.3)$ \\
I don't know &
\end{tabular}

Table 3 Knowledge and prevention measures indexes of the participants regarding Coronavirus Disease 2019 (COVID-19)

\begin{tabular}{ll}
\hline Characteristic & Value \\
\hline Knowledge & \\
Range of index achieved & $12-34$ \\
Mean \pm SD & $24.51 \pm 4.65$ \\
Level of knowledge & \\
Low $(\leq 59 \%) \mathrm{n}(\%)$ & $104(32.2)$ \\
Moderate $(60-79 \%) \mathrm{n}(\%)$ & $170(52.6)$ \\
High $(80-100 \%) \mathrm{n}(\%)$ & $49(15.2)$ \\
Prevention measures & \\
Range of index achieved & $4-16$ \\
Mean \pm SD & $11.39 \pm 2.61$ \\
Level of prevention measures & \\
Low $(\leq 59 \%) \mathrm{n}(\%)$ & $68(21.1)$ \\
Moderate $(60-79 \%) \mathrm{n}(\%)$ & $143(44.3)$ \\
High $(80-100 \%) \mathrm{n}(\%)$ & $112(34.7)$ \\
\hline
\end{tabular}

\section{Other practices and attitudes towards COVID-19}

Around half of the respondents reported treating only dental emergency cases during lockdown (55.7\%), accepting to treat patients with flu like symptoms $(48.6 \%)$ and avoiding treating patients with known or suspected COVID-19 infection (51.7\%). Dentists reported opening their clinics on average of 1.1 days $(\mathrm{SD}=1.3)$ per week during lockdown. Almost all the respondents reported the prescription of paracetamol, 38\% reported the prescription of nonsteroidal anti-inflammatory drugs and only $14 \%$ reported the prescription of corticosteroids for pain management during COVID-19 pandemic.

While $18.6 \%$ of the respondents reported perceiving COVID-19 as very dangerous, $46.4 \%$ and $35 \%$ reported perceiving it as moderately dangerous and not dangerous respectively. Approximately half of the respondents reported that their role in teaching others about COVID19 is very significant and only $12.4 \%$ reported that their role is not significant at all. Lastly, most dentists reported being afraid of getting infected with COVID-19 (66.6\%) and being afraid about family members to get infected due to their occupational exposure (79.3\%).

\section{Factors associated with knowledge towards COVID-19}

The multivariate linear regression model showed that higher knowledge index was associated with working in Mount Lebanon and considering dentist's role important in teaching others about COVID-19. Older age and being self-employed compared to being employed were associated with a lower knowledge index (Table 5a).

\section{Factors associated with high level of prevention measures towards COVID-19}

The multivariate logistic regression model showed that dental specialists excluding surgeons were less likely to have high prevention measures level comparing to general dental practitioners. Besides, participants working in Mount Lebanon were less likely to have high prevention measures level comparing to those working in Beirut. However, dentists living with family members $(\mathrm{OR}=2.3)$ and those who considered dentist's role important in teaching others about COVID-19 $(\mathrm{OR}=2.76)$ were more likely to have high prevention measures level. Lastly, having a higher knowledge index was significantly associated with a higher likelihood of having high prevention measures level $(\mathrm{OR}=1.15)$ (Table $5 \mathrm{~b})$.

\section{Discussion}

In this study, Lebanese dentists achieved an average knowledge index of $65 \%$ and an average prevention measures index of $71 \%$. Scoring high level of prevention measures was associated with higher knowledge index, considering dentists role significant in teaching about COVID-19, living with family members, and being afraid about family members to get infected with the 
Table 4 Prevention Measures adopted by the participating dentists in the dental clinic to prevent the transmission of Coronavirus Disease 2019 (COVID-19)

N (\%)

\begin{tabular}{|c|c|}
\hline \multicolumn{2}{|l|}{ a. Prevention measures adopted in the dental clinic } \\
\hline Use high-speed evacuation (saliva evacuator) for dental procedures producing an droplets or aerosols & $210(65 \%)$ \\
\hline Avoid operations that can produce droplets or aerosols & $186(57.6 \%)$ \\
\hline Frequently clean hands by using alcohol-based hand rub or soap and water & $309(95.7 \%)$ \\
\hline Routinely clean and disinfect surfaces in contact with known or suspected patients & $292(90.4 \%)$ \\
\hline $\begin{array}{l}\text { Use personal protective equipment (such as dental goggles, masks, and gloves) only with patients with known or suspected COVID-19 } \\
\text { infection }\end{array}$ & $301(93.2 \%)$ \\
\hline Avoid moving and transporting patients out of their area unless necessary & $177(54.8 \%)$ \\
\hline All health staff members wear protective clothing & $274(84.8 \%)$ \\
\hline Place patients with known or suspected patients COVID-19 infection in adequately ventilated single rooms & $99(30.7 \%)$ \\
\hline \multicolumn{2}{|l|}{ b. Measures adopted with patients in the dental clinic } \\
\hline Ask patients to sit far from each other & $249(77.1 \%)$ \\
\hline Ask patients to wear masks while in the waiting room & $314(97.2 \%)$ \\
\hline Ask patients to wash hands before getting in the dental chair & $285(88.2 \%)$ \\
\hline Take patient's temperature when they arrive at the clinic & $294(91 \%)$ \\
\hline Screen patients for signs and symptoms of COVID-19 infection & $229(70.9 \%)$ \\
\hline Ask patients about recent travel & $203(62.8 \%)$ \\
\hline Ask patients about recent contact with a person with known or suspected COVID-19 infection & $138(42.7 \%)$ \\
\hline Use a rubber dam & $122(37.8 \%)$ \\
\hline
\end{tabular}

virus. Dental specialists were less likely to achieve high prevention measures index compared to general dental practitioners.

Our multivariate linear model showed that being younger was associated with better knowledge. Findings from other studies showed no association with age [14-16]. First, this difference in results may be partly related to the difference in the questions used to assess dentists' knowledge. Second, we think that Lebanese young dentists were more knowledgeable than older ones because given the lack of national guidelines for dental healthcare in Lebanon before the start of the study, younger dentists who are more familiar with online learning have consulted the international guidelines that have been published on the international health institutions websites earlier since the start of the pandemic. Our study also showed that dentists working in Mount Lebanon region had higher knowledge score than those working in Beirut. This might be related to the significantly younger age of the dentists working in Mount Lebanon comparing to those working in Beirut (mean $=43$ and 48 years old respectively). Lastly, dentists who considered their role significant in teaching others about COVID-19 achieved higher level of knowledge. Although dentists and physicians deliver different type of medical services, their trainings and education share similarities. Therefore, with their knowledge, dental professionals can contribute alongside other healthcare professionals to the fight against COVID-19 pandemic that poses a real challenge to worldwide healthcare systems. For instance, dental professionals should help in patients screening, should develop online platform to spread awareness about COVID-19 and educate the public about oral hygiene especially during lockdown periods.

Our study showed that dentists adopt high level of prevention measures in dental clinics during the pandemic including hand hygiene, surface disinfection, use of PPE and taking patient's temperature as part of the triage process. This practice was similar to previous studies [17-25] and conform with the international $[7,8]$ and LDA guidelines [10]. However Lebanese dentists' prevention practices were suboptimal mainly regarding rubber dam use and patients triage including questions about patient's recent travel and patient's contact with a person with suspected or known COVID-19 infection. Patient triage is crucial in order to identify the patients with suspected or confirmed COVID-19 infection and to schedule the dental care according to the emergency of the patients dental problem and to assess the need in terms of protection equipment and disinfection [10, 26].

Our multivariate logistic regression model showed that better knowledge was associated with having high prevention measures index. To our knowledge, only two previous studies explored the factors associated with prevention practices of dental professionals and reported 
Table 5 Factors associated with knowledge and prevention measures indexes toward Coronavirus Disease 2019 (COVID-19) among participating dentists

Unstandardized Beta $\quad 95 \% \mathrm{Cl}$ of unstandardized Beta

$-0.20 ;-0.09$

$$
-0.15
$$

Age (years)

Work region

Beirut

Mount Lebanon

Others

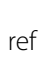

2.66

$-0.44$

$1.54 ; 3.78$

$<0.001$

Employment status

Employee

Self-employed

ref

Un-employed

Dentist's role in teaching others about COVID-19

Not significant

Moderately significant $\quad 2.88$

Very significant

b. Multivariate logistic regression for the factors associated with high prevention measures index (low and moderate combined as a reference)

\section{Work region}

Beirut ref

Others $\quad 0.74$

Mount Lebanon $\quad 0.34$

\section{Specialty}

General practitioner ref

$\begin{array}{ll}\text { Surgeon } & 0.59\end{array}$

Specialist

Living with family members

\section{(2.31}

$1.23 ; 4.34$

0.010

Dentist's role in teaching others about COVID-19

$\begin{array}{ll}\text { Not significant } & \text { ref } \\ \text { Moderately Significant } & 2.76 \\ \text { Very Significant } & 2.13\end{array}$

Afraid about family members to get infected with COVID-19

No ref

Beta regression coefficient, Cl confidence interval, COVID-19 Coronavirus Disease 2019, OR odds ratio

Variables included in the multivariate linear regression model (a): age, years of experience, work region (Others include North of Lebanon, South of Lebanon and Bekaa), occupation, comorbidities, dentist's role about teaching others about COVID-19, afraid of getting infected with COVID-19, afraid about family members to get infected with COVID-19 due to occupational exposure

Variables included in the multivariate logistic regression model (b): gender, work region (Others include North of Lebanon, South of Lebanon and Bekaa), specialty, living with family members, dentist's role about teaching others about COVID-19, afraid of getting infected with COVID-19, afraid about family members to get infected with COVID-19 due to occupational exposure, knowledge index

similar association with knowledge $[19,23]$. Unlike the previous study in Lebanon [23], the present study showed that specialists dentists were less likely to achieve high prevention measures index compared to general dental practitioners. We should note that the previous study was based on a snowball sampling technique and might have suffered from lack of representativeness for dental professionals in Lebanon. Working in Mount Lebanon was 
also associated with lower prevention measures index compared to working in Beirut. This might be explained in part by the younger age of dentists in Mount Lebanon, who may, because of their high knowledge, be more confident when taking more risk regarding the precautionary measures while delivering dental care to their patients. On the other hand, high level of prevention measures practice was associated with considering that dentist's role is significant in teaching about COVID-19, living with family members, and being afraid about family members to get infected with the virus. Like other healthcare professionals, dentists worry that they might carry the virus home to their families. And therefore, they tend to take the essential prevention measures and to adhere to infection control protocol to avoid the transmission of the virus to their families.

On one hand around half of Lebanese dentists in the present study provided emergency treatment for their patients. The percentage of dentists providing emergency interventions was low in India [17, 25] and high in Turkey [15, 21]. This discrepancy might be explained by the availability or the lack of local guidelines and recommendations for dentists regarding the delivery of emergency therapies. In addition, Lebanese dentists opened their clinics on average 1 day per week reflecting a compliance with the total lockdown imposed by the Lebanese authorities. On the other hand, around half of the participants accepted to treat patients with flu like symptoms and patients with known or suspected COVID-19 infection. Most of dentists from previous studies mainly conducted in the early period of the COVID-19 outbreak prefer not to treat patients with COVID-19 [18, 22, 27, $28]$. This was expected as many guidelines stated that only emergency interventions to be performed, with rescheduling of the elective treatment procedures $[7,9]$. These findings may be attributed to the high risk of contamination of different areas of dentist's face during dental practices [29], the inefficacy of standard protective measures in daily clinical work to prevent the spread of COVID-19 [30], the time during which studies were conducted (early during the outbreak), if dental management and infection control guidelines for COVID-19 from dental associations were available and lastly the classification of dentistry as a very high-risk occupation for COVID-19 infection according to the Occupational Safety and Health Act Guidance [31].

More than half of the dentists considered COVID-19 as a dangerous infection and were afraid of getting infected with COVID-19 due to occupational exposure and most of them were afraid about transmitting the virus to their family members. On one hand, there was no effective treatment or vaccine against COVID-19 at the time of the study. On the other hand, the average age for dentists who considered COVID-19 very dangerous was 48 years old in our study compared to an average of 43 years old among those who considered COVID-19 as not dangerous or moderately dangerous. Thus, older dentists are scared from being infected because increased age was shown to be associated with high risk of severe disease and complications [32].

One strength of our study is that it is the first study conducted among dentists in Lebanon with a representative sample randomly selected from the list of dentists registered in the LDA. Besides, the survey collected an important number of variables to identify factors related to knowledge and prevention practices of dentists regarding COVID-19. This study was one of the few that explored the factors related to adherence to prevention measures in dental clinics.

One limitation of this study is the lack in the knowledge questionnaire of questions related to the type of masks used, the use of mouthwash before and/or during dental care delivery and the choice of disinfectant solutions. Other limitations included recall and information bias related to the use of an online self-administered questionnaire as survey instrument.

\section{Conclusions}

As information about COVID-19 continues to evolve rapidly, further improvement and update would be beneficial to optimally manage patients in this challenging situation. The knowledge on infection control in dental clinics should be strengthened through online educational trainings if physical meetings are not possible due to recurrent lockdowns. National guidelines for dental care should be regularly updated and shared with dentists particularly older ones.

\section{Abbreviations}

COVID-19: Coronavirus disease 2019; LDA: Lebanese Dental Association; PPE: Personal protective equipment; SARS-CoV-2: Severe acute respiratory syndrome coronavirus 2; SD: Standard deviation.

\section{Acknowledgements}

Not applicable

\section{Authors' contributions}

M.H. and N.S. conceptualized and designed the study, C.H., N. H. and M. C. collected all the data and contributed to the analysis of the data, M.H. and N.S. interpreted the results, M.H., C.H., N. H. and M. C. prepared the first draft of the manuscript, M.H. and N.S. reviewed and edited the final draft of the manuscript, All authors read and approved the final manuscript.

\section{Funding}

No funding was received.

\section{Availability of data and materials}

The datasets generated and analyzed during the current study are not publicly available due to limitations of ethical approval involving the patient data and anonymity but are available from the corresponding author on reasonable request. 


\section{Declarations}

\section{Ethical approval and consent to participate}

The survey was completely voluntary and anonymous. Confidentiality of the responses was guaranteed to the participants. Participant's informed consent was obtained before the completion of the questionnaire. The study being observational and respecting participants' anonymity and confidentiality, the institutional review board of the Lebanese university waived the need for an official approval.

\section{Consent for publication}

Not applicable.

\section{Competing interests}

The authors declare that they have no competing interests.

\section{Author details}

${ }^{1} \mathrm{CERIPH}$, Center for Research in Public Health, Pharmacoepidemiology Surveillance Unit, Faculty of Public Health II, Lebanese University, Fanar, Lebanon. ${ }^{2}$ INSPECT-LB: Institut National de Santé Publique, Epidémiologie Clinique Et Toxicologie - Liban, Beirut, Lebanon. ${ }^{3}$ Faculty of Public Health, Lebanese University, Fanar, Lebanon.

Received: 5 March 2021 Accepted: 27 April 2021

Published online: 06 May 2021

\section{References}

1. Cucinotta D, Vanelli M. WHO Declares COVID-19 a Pandemic. Acta Biomed. 2020;91(1):157-60

2. Wang C, Horby PW, Hayden FG, Gao GF. A novel coronavirus outbreak of global health concern. The Lancet. 2020;395(10223):470-3.

3. World Health Organization. Weekly epidemiological update - 9 February 2021 [Internet]. 2021 [cited 2021 Feb 10]. Available from: https://www. who.int/publications/m/item/weekly-epidemiological-update---9-febru ary-2021

4. Republic of Lebanon, Ministry of Public Health. Novel Coronavirus 2019 [Internet]. 2021 [cited 2021 Feb 17]. Available from: http://www.moph. gov.lb/en/Media/view/46717/novel-coronavirus-2019

5. Stasi C, Fallani S, Voller F, Silvestri C. Treatment for COVID-19: an overview. Eur J Pharmacol. 2020;889:173644

6. Seneviratne CJ, Lau MWJ, Goh BT. The role of dentists in COVID-19 is beyond dentistry: voluntary medical engagements and future preparedness. Front Med (Lausanne). 2020;7:566.

7. CDC Releases Interim Reopening Guidance for Dental Settings [Internet]. 2020 [cited 2020 Nov 24]. Available from: https://www.cdc.gov/oralh ealth/infectioncontrol/statement-COVID.html

8. CDC Guidance for Dental Settings Echoes ADA Guidance [Internet]. 2020 [cited 2021 Feb 7]. Available from: https://www.ada.org/en/press-room/ news-releases/2020-archives/may/cdc-guidance-for-dental-settingsechoes-ada-guidance

9. ADA recommending dentists postpone elective procedures [Internet]. 2020 [cited 2020 Nov 25]. Available from: https://www.ada.org/en/publi cations/ada-news/2020-archive/march/ada-recommending-dentistspostpone-elective-procedures

10. El Hayeck E, Al Asmar B, Anka C. SARS-CoV-2 and dental practice [Internet]. Lebanese Dental Association. 2020 [cited 2021 Feb 4]. Available from: http://www.lda.org.lb/uploaded/editorimages/file/2020/SARS-CoV2\%20AND\%20DENTAL\%20PRACTICE.pdf

11. Mansour H, Feghali M, Saleh N, Zeitouny M. Knowledge, practice and attitudes regarding antibiotics use among Lebanese dentists. Pharm Pract (Granada). 2018:16(3):1272.

12. Nur Ain M, Azfar M, Omarulharis S, Azfar H, Maryam A, Hafizah S, et al. Knowledge, attitude and practice of dengue prevention among sub urban community in Sepang, Selangor. Int J Public Health Clin Sci. 2017:4(2):73-83

13. Bouyer J, Hermon D, Cordier S, Derriennic F, Stucker I, Stengel B, et al. Épidémiologie: principes et méthodes quantitatives. Paris: Lavoisier; 2009. p. 515.
14. Kinariwala N, Samaranayake L, Perera I, Patel Z. Knowledge, Awareness and Perceptions of Coronavirus Disease 2019 (COVID-19) in a Cohort of Indian Dental Professionals: A Questionnaire-Based Study. 2020 Preprints. doi: https://doi.org/10.20944/preprints202005.0442.v1.

15. Sezgin GP, ŞirinoĞlu Çapan B. Assessment of dentists' awareness and knowledge levels on the Novel Coronavirus (COVID-19). Braz Oral Res. 2020;34:e112.

16. Sarfaraz S, Shabbir J, Mudasser MA, Khurshid Z, Al-Quraini AAA, Abbasi MS, et al. Knowledge and attitude of dental practitioners related to disinfection during the COVID-19 pandemic. Healthcare. 2020;8(3):232.

17. Singh Gambhir R, Singh Dhaliwal J, Aggarwal A, Anand S, Anand V, Kaur BA. Covid-19: a survey on knowledge, awareness and hygiene practices among dental health professionals in an Indian scenario. Rocz Panstw Zakl Hig. 2020:71(2):223-9.

18. Khader Y, Al Nsour M, Al-Batayneh OB, Saadeh R, Bashier H, Alfaqih M, et al. Dentists' Awareness, Perception, and Attitude Regarding COVID-19 and Infection Control: Cross-Sectional Study Among Jordanian Dentists. JMIR Public Health Surveill. 2020;6(2):e18798.

19. Kamate SK, Sharma S, Thakar S, Srivastava D, Sengupta K, Hadi AJ, et al. Assessing knowledge, attitudes and practices of dental practitioners regarding the COVID-19 pandemic: a multinational study. Dent Med Probl. 2020:57(1):11-7.

20. Tarakji B, Nassani MZ, Alali FM, B Alsalhani A, Alqhtani NR, Bin Nabhan A, et al. COVID-19-Awareness and Practice of Dentists in Saudi Arabia. Int J Environ Res Public Health. 2021;18(1).

21. Karayürek F, Yilmaz Çırakoğlu N, Gülses A, Ayna M. Awareness and Knowledge of SARS-CoV-2 Infection among Dental Professionals According to the Turkish National Dental Guidelines. Int J Environ Res Public Health. 2021;18(2).

22. Mustafa RM, Alshali RZ, Bukhary DM. Dentists'knowledge, attitudes, and awareness of infection control measures during COVID-19 outbreak: a crosssectional study in Saudi Arabia. Int J Environ Res Public Health. 2020;17(23)

23. Nasser Z, Fares Y, Daoud R, Abou-Abbas L. Assessment of knowledge and practice of dentists towards Coronavirus Disease (COVID-19): a crosssectional survey from Lebanon. BMC Oral Health. 2020;20(1):281.

24. Tokuc B, Coskunses FM. Knowledge, attitude and practice of dentists in Coronavirus disease 2019 pandemic in Turkey. Eur Oral Res. 2020;54(2):86-91.

25. Teja KV, Vasundhara KA, Sriram G. Knowledge, Awareness, and Practice of Dentists in Preventing Novel Corona Virus (COVID-19) Transmission - A Questionnaire Based Cross-Sectional Survey. Braz Dent Sci. 2020;1-9.

26. Ather A, Patel B, Ruparel NB, Diogenes A, Hargreaves KM. Coronavirus Disease 19 (COVID-19): implications for clinical dental care. J Endod. 2020;46(5):584-95.

27. Shahin SY, Bugshan AS, Almulhim KS, AISharief MS, AI-Dulaijan YA Siddiqui I, et al. Knowledge of dentists, dental auxiliaries, and students regarding the COVID-19 pandemic in Saudi Arabia: a cross-sectional survey. BMC Oral Health. 2020;20(1):363.

28. Bakaeen LG, Masri R, AlTarawneh S, Garcia LT, AlHadidi A, Khamis AH, et al. Dentists' knowledge, attitudes, and professional behavior toward the COVID-19 pandemic: a multisite survey of dentists' perspectives. J Am Dent Assoc. 2021;152(1):16-24

29. Nejatidanesh F, Khosravi Z, Goroohi H, Badrian H, Savabi O. Risk of contamination of different areas of dentist's face during dental practices. Int J Prev Med. 2013;4(5):611-5.

30. Meng L, Hua F, Bian Z. Coronavirus Disease 2019 (COVID-19): Emerging and future challenges for dental and oral medicine. J Dent Res. 2020;99(5):481-7.

31. OSHA. Guidance on Preparing workplaces for COVID-19 [Internet]. 2020 [cited 2021 Feb 6]. Available from: https://www.osha.gov/pls/publicatio ns/publication.searchresults?pSearch=Guidance+on+Preparing+ Workplaces+for+COVID-19

32. Liu K, Chen Y, Lin R, Han K. Clinical features of COVID-19 in elderly patients: a comparison with young and middle-aged patients. J Infect. 2020;80(6):e14-8.

\section{Publisher's Note}

Springer Nature remains neutral with regard to jurisdictional claims in published maps and institutional affiliations. 